\title{
Estimating the available bandwidth for real-time traffic over best effort networks
}

\author{
F. Davoli, O. Khan, P. Maryni \\ Department of Communications, Computer and System Sciences \\ DIST-University of Genoa \\ Via Opera Pia 13, 16145 Genova, Italy \\ Tel. +3910 3532732, Fax. +3910 3532948, \\ email:franco@dist.unige.it
}

\begin{abstract}
A mechanism for the estimation of the available bandwidth between two end-points of a best-effort network is presented. The estimation is obtained by a simple statistical analisys of the effect which the network has on a synchronous train of packets. The possibility of exploiting self-similar characteristics of the delay jitters is also discussed.
\end{abstract}

Keywords: multimedia, load estimation, self-similarity.

\section{INTRODUCTION}

Distributed multimedia applications are characterized by the presence a mix of heterogeneous traffic flows (e.g., video, voice, data) with different statistical features and performance requirements. In this context, a number of services may require a very large bandwidth and stringent Quality of Service (QoS) constraints. This does not constitute a problem in the presence of high-performance networks (e.g., ATM), where the availability of large bandwidth and the presence of a number of control mechanisms can guarantee a wide range of QoS. Hovewer, there are still several situations (which are likely to last in the medium term), where the presence of networks with either limited capacity or "best-effort" service type may represent a potential bottleneck.

As a matter of fact, the widespread diffusion of the Internet Protocol Suite (based on a best-effort service type) determines the development of multimedia applications which rely upon protocols with almost no QoS guarantees (e.g., UDP and IP), in both LAN and WAN frameworks. It is likely that such applications will continue to grow in the medium term and to receive a further thrust from the increase in the network capacity (e.g., IP over ATM).

This scenario suggests that the multimedia environment will be characterized by a wide variety of traffic types and by the presence of networks with possibly very different capabilities. In particular, some 
traffic types, like voice and video, will require QoS guarantees (e.g., in terms of maximum delay, delay jitter and throughput), whereas some networks will offer an asynchronous service which would be unable to ensure the required QoS. Control procedures should therefore be envisaged (like control of admission, congestion and flow), capable of operating at the edges of the best-effort networks, in a way not too dissimilar from the analogous ones performed at a lower architectural level for the ATM network.

In this paper we address a problem which is strictly related to the implementation of such control functions, namely the estimation of the "available bandwidth" between two end-points of a best-effort network.

By available bandwidth we mean here a quantity which makes it possible to estimate whether a given telecommunication service can be set up between two end-points of a network.

The paper is organized as follows. The next Section describes the system model. The statistical analysis which leads to the estimation mechanisms is described in Section 3. In Section 4 we discuss some issues related with a self-similar approach and in Section 5 we draw the conclusions.

\section{THE SYSTEM MODEL}

Consider two end points of a channel of a generic network, one of them acting as a transmitter while the other one acting as a receiver. We may think of a channel as a link of a network or a path composed of more than one link. The transmitter generates and sends a short packet to the receiver once every $T$ seconds. The receiver, upon reception of a packet, computes its interarrival time, which will be used for an estimate of the load of the channel. In such a framework, if the channel is lightly loaded, it is reasonable to expect that also the receiver will receive a synchronous (or quasi-synchronous) train of packets. On the other hand, as the load of the channel increases, we expect to see, at the receiver site, a degradation of such a synchronicity.

Figure 1 gives a pictorial representation of this simple model scheme.

These measure packets do not need to carry information and, therefore, their size can be very small. Indeed, if $T$ is chosen properly, the train of packets does not significantly alter the overall load of the channel.

This way of estimating the load has several advantages. First, there is no need of polling the nodes of the channel (e.g., routers) in order to get load information: not everyone, in fact, can normally access routers and, when access rights are given, routers might have different access protocols (e.g., SNMP, CMIP). Second, this way of estimating does not require to know almost anything about the channel. In particular, we do not need to know the number and type of active connections on the channel in order to come up with an estimation. This estimating mechanism provides a way of measuring directly the part of the channel still available, the free part of it.

Each valuable real time service (e.g., video, voice) has the need of a certain degree of synchronicity as a main Quality of Service requirement. By mapping the degree of synchronicity of the train of packets at the receiver for each service, we are able to say whether such a service can fit into the channel or network.

A further study, beyond the scope of this work, is to map a connection of a given type with a level of degradation of the synchronicity of the train of packets. With such a further mapping we can say how many connections of a given type could fit into a channel.

It is worth noting that not every source-destination pair needs generating the test train of packets (this procedure would be likely to generate an excessively high load in the presence of a large number of connections, even though the length of a test packet is of the order of few bytes). One possible alternative is to have a pair of "multimedia servers" performing this operation and distributing the results of the 


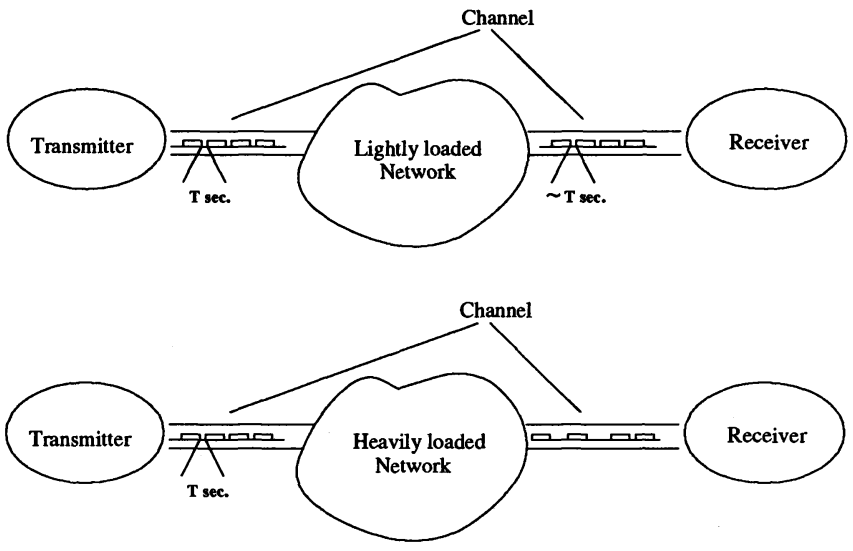

Figure 1 The system model.

analysis, either periodically (with period much larger than $T$ ) or on demand. In this case, the additional load caused by the measurement packets could really be considered as negligible. The presence of such "network elements" performing the estimation (in lieu of the sources themselves) would also have the advantage of by-passing possible source control mechanisms that might alter the statistics (e.g., traffic shaping or policing).

\section{THE ESTIMATING MECHANISM}

The aim of the Estimating Mechanism is to come out with a measure of the level of synchronicity of the train of packets at the receiving end (Bolot 1993). Figure 2 shows the interarrival times relative to an experiment over a "channel" composed of two Ethernet LANs linked, through routers, by a $1.5 \mathrm{Mbit} / \mathrm{s}$ serial line, with $T=10 \mathrm{~ms}$. For the first 50 seconds of the experiment (i.e., 5000 packets), the channel is lightly loaded and then a heavy $f t p$ data transfer takes place. The effect of the heavy data transfer on the train synchronicity can be easily seen: before the $f t p$ takes place, data are gathered around the value of $T$; immediately after the $f t p$, the presence of bursts of packets is demonstrated by the quite large number of data close to zero.

Let us denote with $x_{k}$ the time lag between the arrival instants of packets $k$ and $k-1$, and let us consider first the interarrival time average. More specifically we focus on the average $\bar{x}_{m}(i)$ computed over a window of $m$ time lags,

$\bar{x}_{m}(i)=\frac{1}{m} \sum_{k=m * i}^{m *(i+1)} x_{k}$ 


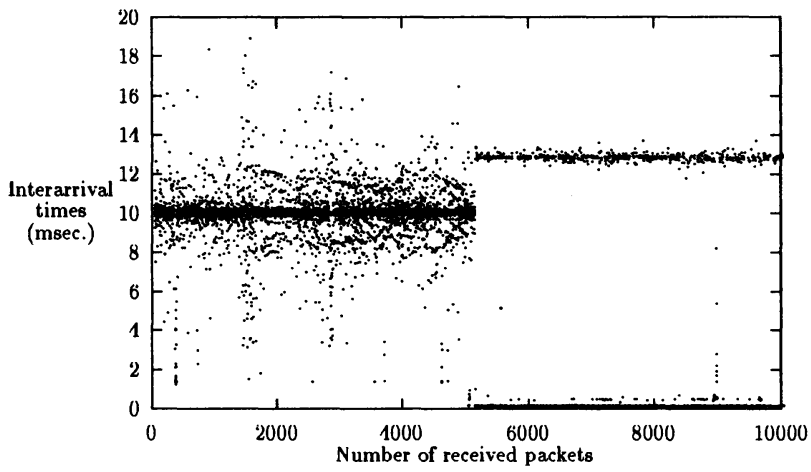

Figure 2 Interarrival times seen by the receiver.

Figure 3 shows this value computed over a window of 10 time lags (i.e., $m=10$ ). The effect of the $f t p$ connection is evident: averages are much more spread around the value of $T$.

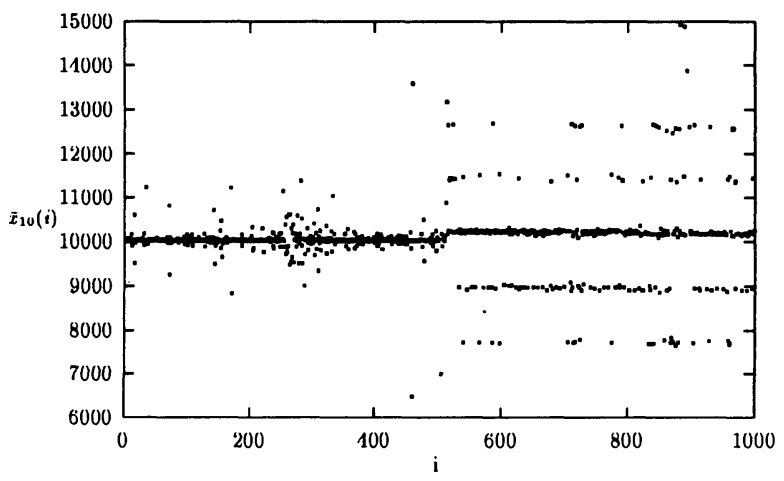

Figure 3 "Sliding" averages $(m=10)$.

The following step is to look at the standard deviation. More in particular, we considered a "sliding" standard deviation estimator defined as follows:

$\sigma_{m}(i)=\sqrt{(1 /(m-1)) \sum_{j=m * i}^{m *(i+1)}\left(x_{j}-\bar{x}_{m}(i)\right)^{2}}$ 
Figure 4 shows standard deviations $\sigma_{10}(i)$, computed over a window of 10 time lags: the presence of the $f t p$ connection is also evident in this figure.

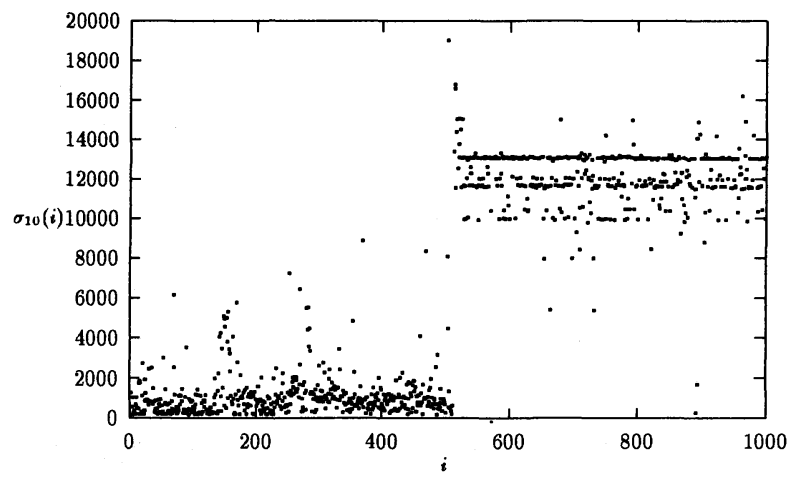

Figure 4 "Sliding" standard deviation $(m=10)$.

Unfortunately, time lags, along with sliding averages and standard deviations only give a good "pictorial" feeling of the degree of asynchronicity. In other words, they do not give a stable value for a reliable measure of the degree of asynchronicity. To see this, let us consider another experiment. In this second experiment, monitoring is performed on the same channel but, this time, no particular load situation is imposed on it: measurements have been taken during a normal working period. During this experiment, 9000 packets have been involved in the measurements. If we look at the values of $x_{m}$, even for different values of $m$, we realize that, even if the value of $T$ is persistent, the plot is everything but stable. Figures $5(\mathrm{a}, \mathrm{b})$ show this point quite clearly.

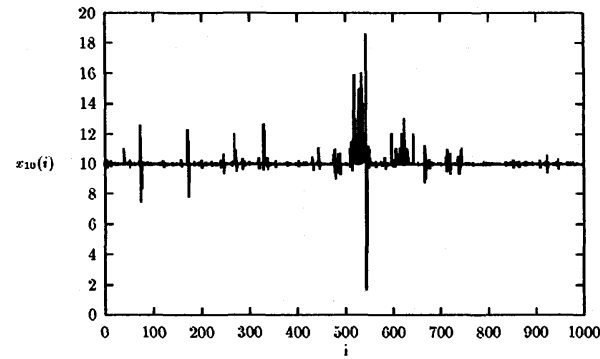

(a)

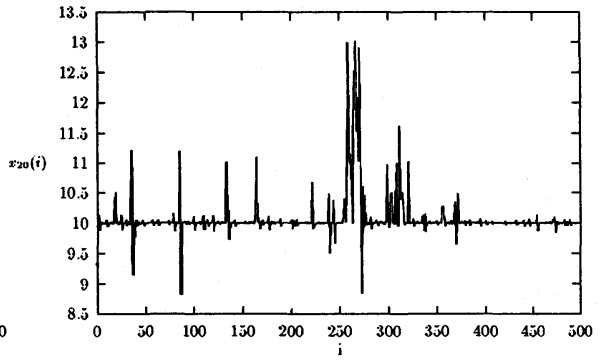

(b)

Figure 5 Sliding averages for the second experiment. (a) $m=10$; (b) $m=20$.

In order to get a stable value, we considered the $\epsilon$-percentile $\xi_{\epsilon, n}(i)$ computed on a window of $n$ sliding 


\begin{tabular}{lc}
\hline situation & $\xi_{0.3,10}$ \\
\hline empty & 1 \\
nv & 5 \\
ftp & 12 \\
\hline
\end{tabular}

Table 1 Percentile measurements in different situations.

variances, where $\xi_{\epsilon, n}(i)$ is such that on the set $\left[\sigma_{m}(n * i), \ldots, \sigma_{m}(n *(i+1)]\right.$, less than $100 \epsilon \%$ of the values are smaller than $\xi_{\epsilon, n}(i)$. This looks like having all the nice characteristics we are looking for: it is easy to compute, it gives a stable load estimate and it is also quite fast in reacting to varying load conditions.

Figure 6 shows the values of $\xi_{\epsilon, n}(i)$ for the first experiment. We set $\epsilon=0.3, n=30$, and $m=10$ (these figures work well over a large number of experiments). Hence, each value corresponds to the 0.3percentile computed on a set of 30 sliding standard deviations which, in turn, are computed over 10 time lags. Notice that the plot abruptly changes after $i=17$ : this value corresponds to the 5100-th time lag (i.e., $17 \cdot 30 \cdot 10$ ), which approximately is the time the $f t p$ connection takes place.

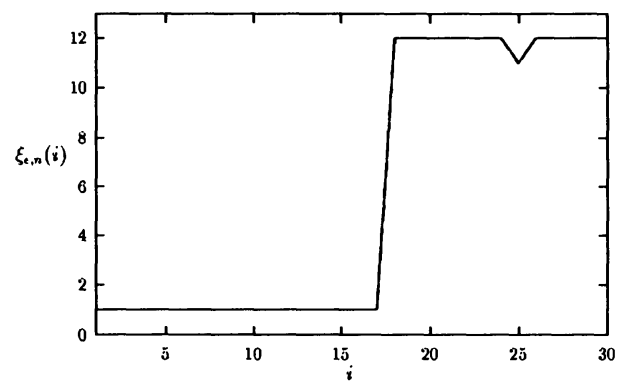

Figure 6 Plot of $\xi_{\epsilon, n}(i), m=10, n=30, \epsilon=0.3$.

Table 1 shows measured values of $\xi_{0.3,10}$ in three different situations (those situations have been tried with no other users on the channel). The first one is the situation with no transmission at all on the channel; the second one is the popular "nv" application, which is a tool for sending a video signal to a remote workstation with different levels of quality; the third one is a plain " $\mathrm{ftp}$ " connection along the channel.

These measurements can be utilized for an estimate of the ability of the network to satisfy the QoS for a given application. For example, we noticed that a "nv" connection cannot be run on that channel at its best quality (in terms of frame rate and/or window dimension) if an "ftp" is running at the same time. Hence, we know that if the measured value of $\xi_{0.3,10}$ is greater than 12 then no "nv" at its best quality is possible. We fixed three different levels of quality: a best one, a medium one and a sufficient one. In 


\begin{tabular}{lc}
\hline \multicolumn{1}{c}{ level } & $\xi_{0.3,10}$ \\
\hline best & $<12$. \\
medium & $<20$ \\
sufficient & $<25$ \\
\hline
\end{tabular}

Table 2 Grades needed for the three different levels of quality.

this framework, the terms "best", "medium" and "sufficient" have to be intended as Perceived Quality of Service (PQoS) (Seiz 1994, Ferrari 1990). Table 2 shows the requirements for these three levels of quality.

\section{HOW ABOUT SELF-SIMILARITY ?}

Figure 7(a) shows the values of interarrival times for the second experiment we presented in this paper (the one Figure 5 refers to). The plots (b), (c), and (d) represent portions of plot (a) showed over different time scales. This suggests that a synchronous train of packets, when transferred over an asynchronous channel, gets to the receiver with interarrival times which seem to look like having self-similar characteristics. Many examples can be found in literature which discuss self-similarity both in general terms (see for example Mandelbrot 1983) and within telecommunication systems (see for example Leland 1994, Erramilli 1993, Erramilli and Willinger 1993, Erramilli 1996).

Another possible direction is, therefore, to exploit self-similarity. We considered the so-called R/S analysis because of its nice property, among others, of being simple and straightforward. Given a collection of samples $x_{i}, i=1, \ldots, N$, the let us define $R S(d)=R(d) / S(d), d<N$, where:

$$
\begin{aligned}
& R(d)=\max _{1 \leq k \leq d}\left[\sum_{i=1}^{k}\left(x_{i}-\bar{x}(d)\right)\right]-\min _{1 \leq k \leq d}\left[\sum_{i=1}^{k}\left(x_{i}-\bar{x}(d)\right)\right], \\
& S(d)=\sqrt{\frac{1}{d-1} \sum_{j=1}^{d}\left(x_{j}-\bar{x}(d)\right)},
\end{aligned}
$$

and

$\bar{x}(d)=(1 / d) \sum_{i}^{d} x_{i}$.

$R / S$ analysis is generally utilized to test for self-similarity of a set of collected data. The variable $d$ can be interpreted as a time lag and the function $R / S$ is able to capture persistence on different time scales, if any. Following this analysis, if data are characterized by self-similarity, then the function $R S(d)$ 


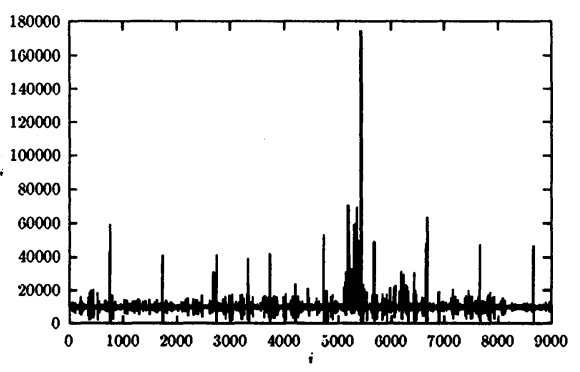

(a)

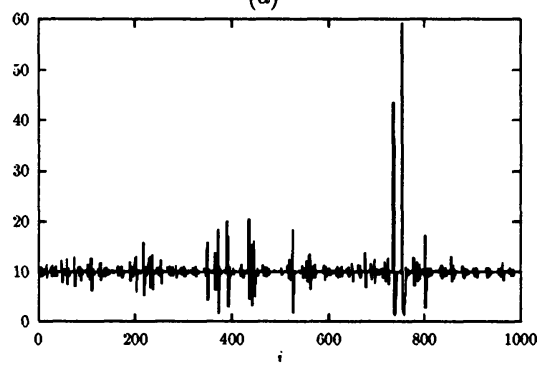

(c)

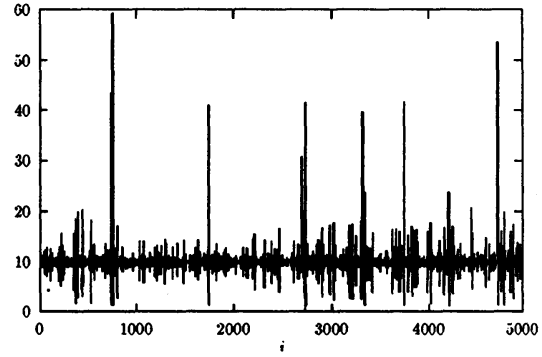

(b)

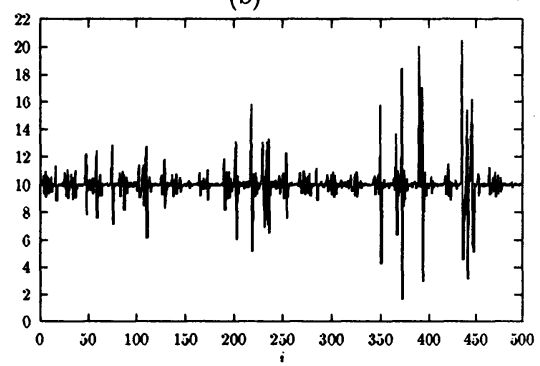

(d)

Figure 7 Time lags over different time scales. (a) 9 sec. ; (b) 5 sec. ; (c) 1 sec. ; (d) 0.5 sec.

should show an asymptotic slope (the so-called "Hurst Effect") which approximates $d^{H}(0.5 \leq H \leq 1)$. $H$ is called "Hurst parameter" (Leland 1994). The $R / S$ analysis of the second experiment is shown in Figure 8. In the Figure it is also shown that, for that data, the Hurst parameter $H$ is approximately 0.7 .

Another powerful analysis is the so-called " $\operatorname{Var}\left(X^{m}\right)$ " one. This considers the variance of the series $X^{m}(k)=m^{-1}\left(X_{k m-m+1}+\ldots+X_{k m}\right)$, with $k \geq 1$. A long-range dependence, or heavy tail, can be detected by plotting $\operatorname{Var}\left(X^{m}\right)$ versus $m$ on a log-log scale and looking for an asymptotic slope $m^{-\beta}$ with $0<\beta<1$. It can be shown that $H=1-\beta / 2$, where $H$ is the Hurst parameter of the $R / S$ analysis.

Figure 9 shows the plot of $\log \operatorname{Var}\left(X^{m}\right)$ versus $\log m$ in the three cases (i.e., empty, nv and ftp). As regards the asymptotic slope, $\beta$ is about -0.75 . Notice that the parameter $\beta$ is sensitive to the load conditions. This suggests that also self-similar techniques could be utilized for load estimates. Unfortunately, self-similar parameters usually represent asymptotic behaviours. In other words, when it comes to exploit self-similar analysis, we have to remember that many time scales are involved and that, therefore, we need to analyze a large number of samples. Hence, this techniques are scarcely applicable in a real-time framework, where the estimating mechanism must be fastly reactive to load variations. 


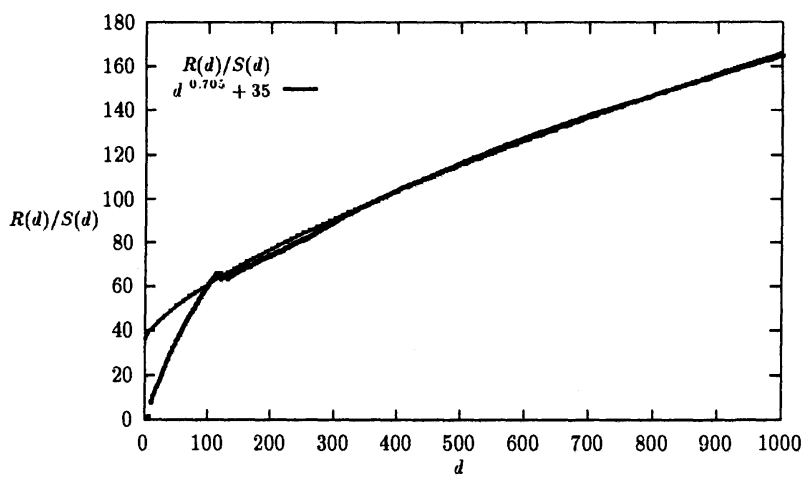

Figure 8 Hurst effect with $R / S$ plot.

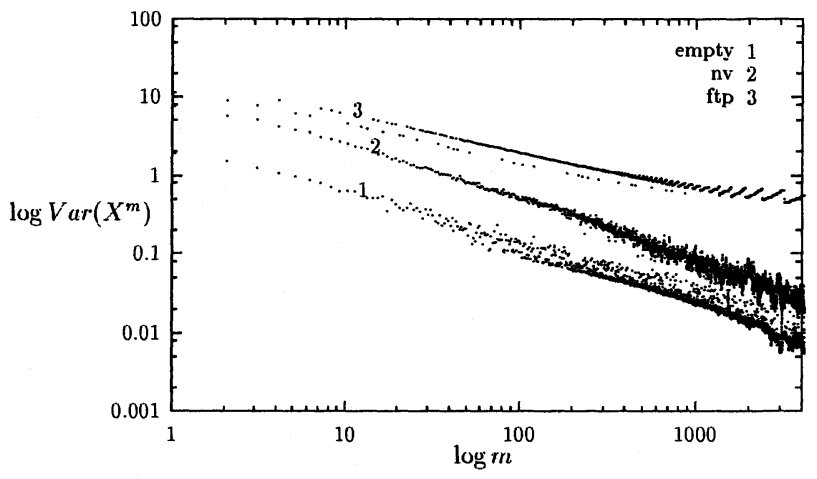

Figure 9 The $\operatorname{Var}\left(X^{m}\right)$ analysis.

\section{CONCLUSIONS}

A mechanism for estimating the available bandwidth between two end points of a best-effort network has been presented. The purpose of this mechanism is to carry out the estimation procedure with no information the overall offered load. Focus has been primarily put on real-time services even though also non real-time ones often have maximum delay requirements. The mechanism works by performing, at one side of the network, a set of statistical operations on the measured delay of a train of packet synchronously transmitted by the other end point.

As regards the possible applications of the scheme, we can mention two cases. One possibility is to use the information on the available bandwidth to control the rate of real-time sources (e.g., video), e.g., by 
decreasing it equally whenever a threshold is exceeded. A second one is in admission control; in this case, however, one should keep in mind that tere is a certain amount of uncontrollable "background traffic" (e.g., ftp), which might produce significant changes after a connection admission/refusal decision has been taken. The two mechanisms might be employed jointly. This will be the subject of future work.

\section{REFERENCES}

JC. Bolot. Characterizing End-to-end Packet Delay and Loss in the Internet. Journal of High-Speed Networks, vol. 2, no. 3, pages 305-323, December 1993.

N. Seiz, S. Wolf, S. Voran, and R. Bloomfield. User-oriented Measures of Telecommunication Quality. IEEE Communications Magazine, pages 56-66, January 1994.

D. Ferrari. Client Requirements for Real-Time Communication Services. IEEE Communications Magazine, pages 65-72, November 1990.

W.E. Leland, M.S. Taqqu, W. Willinger, and D.V. Wilson. On Self Similar Nature of Ethernet Traffic (Extended Version). In IEEE Transactions on Networking, pages 1-15, February, 1994.

A. Erramilli. Chaos, Fractals and Traffic. In Proceedings of the 6th MultiG Workshop, Electrum, StockolmKista, May 1993.

A. Erramilli e W. Willinger. Fractal Properties in Packet Traffic Measurements. In Proceedings of ITC Seminar, St. Petersburg, 1993.

A. Erramilli, O. Narayan, and W. Willinger. Experimental queueing analysis with long-range dependent packet traffic. IEEE Transactions on Networking, pages 209-222, April 1996.

B. Mandelbrot. The Fractal Geometry of Nature. Freeman, New York, 1983. 